\title{
Too large to fit? Recent developments in macromolecular imprinting
}

\author{
Yi Ge and Anthony P.F. Turner \\ Cranfield Health, Cranfield University, Silsoe, Bedfordshire, MK45 4DT, UK
}

\begin{abstract}
Molecular imprinting involves the synthesis of polymers in the presence of a template to produce complementary binding sites with specific recognition ability. The technique has been successfully applied as a measurement and separation technology, producing a uniquely robust and antibody-like polymeric material. Low molecular weight molecules have been extensively exploited as imprint templates, leading to significant achievements in solid-phase extraction, sensing and enzyme-like catalysis. By contrast, macromolecular imprinting remains underdeveloped, principally because of the lack of binding site accessibility. In this review, we focus on the most recent developments in this area, not only covering the widespread use of biological macro-templates but also highlighting the emerging use of synthetic macro-templates, such as dendrimers and hyperbranched polymers.
\end{abstract}




\section{Introduction}

Molecular recognition is fundamental to the function of biological systems and has great practical importance in advancing science and technology. Among the various existing approaches to produce biomimetic systems for molecular recognition, 'molecular imprinting' has delivered several distinct advantages and broad applications [1]. In general terms, molecular imprinting can be described as the formation of a polymer network with specific recognition and binding sites that are complementary to a template, which could be either a biomolecule or a synthetic compound. During this formation, the functional monomers are polymerised in the presence of a template, which is subsequently removed by washing and/or extraction after the polymerisation. Thus, the re-binding template could be recognised by the polymer. The product of molecular imprinting is called a molecularly imprinted polymer (MIP) (see Figure 1). Generally, there are three approaches to molecular imprinting, and these can be distinguished in terms of the interaction between the template molecule and the functional monomer. They are covalent molecular imprinting, non-covalent molecular imprinting and metal-coordinating molecular imprinting.

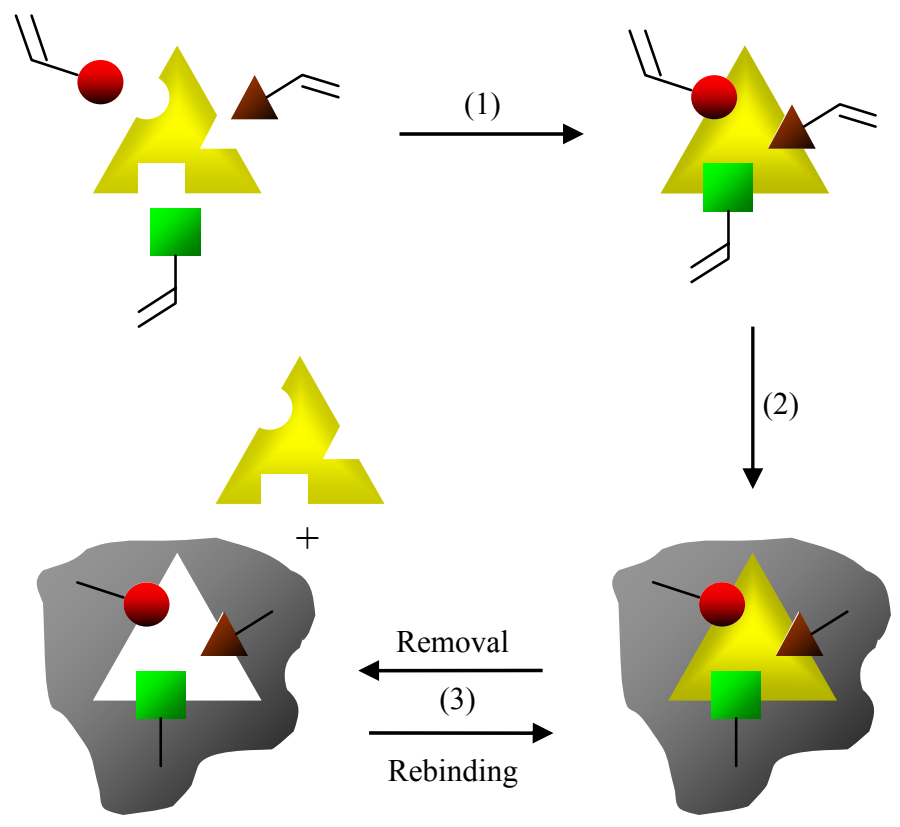

Fig. 1 Molecular imprinting: (a) Formation of a pre-polymerisable complex with template (yellow) and functional monomers (red, green and brown) by interactions (non-covalent, covalent and/or metal coordination interactions) that occur between complementary functionalities in the template molecule and functional monomer units. (b) Polymerisation with excess cross-linking agent to produce the MIP (grey); (c) Template (yellow) removal by washing and/or extraction, which leaves specific recognition sites that are complementary to the templates in terms of size, shape and chemical functionality orientations, thus enabling subsequent recognition of the template during the rebinding process.

MIPs are now well known as synthetic polymeric receptors or robust artificial antibodies ('plastibodies') and have attracted considerable attention from the scientific and industrial communities owing to their inherent simplicity, reusability, robust polymer network and cost-effectiveness [1,2]. In the past five years, a large number of publications have reported imprinting of relatively small molecules such as sugars, steroids, pesticides, certain drugs and amino acid derivatives. This demonstrates that MIPs are capable of highly specific recognition of templates and can be used in preparative and analytical separations, solid-phase extractions, chemical sensors, enzyme-like catalysts, drug delivery and library screening tools, etc [3-7]. By contrast, however, the technology for large-molecule imprinting, particularly macromolecular imprinting, remains underdeveloped.

A macromolecule, such as a polymer or protein, consists of many smaller structural units covalently linked together [8]. Interest and demand for macromolecular recognition is continually growing to further exploit natural systems for the control of biological form and function, particularly for biomedical and biodiagnostic applications. In a conventional MIP, the small template molecule is able to move freely (i.e. to reach or leave) to the complementary binding sites in the MIP matrix. However, it 
is more difficult for a macromolecular template to penetrate the polymer network to access the binding pockets. Furthermore, the increased structural complexity and large size of a macromolecule leads to more non-specific and heterogeneous binding sites, and as a result the MIPs display poor recognition behaviour. Additionally, owing to the complex properties of some biomacromolecules, such as proteins and cells, the conditions of biomacromolecular imprinting need to be close to their natural environment to ensure that their conformational integrities and binding activities are maintained.

Protein imprinting is the most intensively studied area of macromoleculular imprinting, and work up to 2005 has been extensively reviewed [9-11]. This review therefore focuses on the most recent developments using biological templates, including proteins and other biomacromolecules, and in particular we reveal the emerging area of synthetic macrotemplates, such as dendrimers and hyperbranched polymers.

\section{Biomacromolecular imprinting}

Protein imprinting

As one of the most important biomacromolecules, proteins have been extensively investigated as imprint templates since molecular imprinting technology was established more than 35 years ago [911], but progress has been slow. Unsurprisingly, the bottleneck for this technology is the natural properties of proteins. Proteins are biomacromolecules with molecular weights ranging from $6000 \mathrm{Da}$ to several million Da. This large size hinders the protein from both reaching and leaving artificial binding sites. There are also a large number of potential binding sites and functional groups on the surface of proteins, and this complexity increases the probability that non-specific interactions will occur between proteins and MIPs, leading to poor selectivity and cross reactivity. More importantly, proteins are often incompatible with the organic solvents used in the synthesis of most MIPs. The use of an aqueous solution for the molecular imprinting process greatly restricts the choice of reactive functional monomers and cross-linkers. Moreover, it has been pointed out that water could compete for and potentially disrupt any hydrogen bonds between the template and the functional monomers [12]. Given that hydrogen bonds play a significant role in non-covalent molecular imprinting, which is the most employed and practical approach, water is far from the ideal solvent. In addition to being incompatible with solvents, proteins are sensitive to temperature, $\mathrm{pH}$ and ionic strength, extremes of which can result in denaturation, conformational changes or aggregation. All the above factors, together with the high costs of synthesizing some pure proteins, make the route to effective and practical protein imprinting tortuous.

Approaches to protein imprinting can generally be classified into three categories: bulk imprinting, surface imprinting and epitope imprinting, depending on the imprinted part or fragment of protein template (i.e. whole protein, partial protein or a small epitope part of protein) (see Box 1 and Table 1).

Bulk imprinting: The protein template is wholly imprinted in the bulk of the polymer matrix, and is recognised and bound as a whole molecule by functional monomers.

Surface imprinting: The protein template is partially imprinted in the bulk of polymer matrix, and the recognition and binding sites are situated at or close to the surface of MIP.

Epitope imprinting: Only a small epitope part of protein is imprinted as the template, but the resulting MIP is able to recognise the whole protein.

Box 1. Characteristics of the three main approaches forprotein imprinting

\begin{tabular}{|l|l|l|l|l|}
\hline $\begin{array}{l}\text { Imprinting } \\
\text { approach }\end{array}$ & $\begin{array}{l}\text { Protein/epitope } \\
\text { template }\end{array}$ & $\begin{array}{l}\text { Interaction between } \\
\text { functional } \\
\text { monomer(s) and } \\
\text { template }\end{array}$ & $\begin{array}{l}\text { Polymer } \\
\text { composition }\end{array}$ & Ref \\
\hline $\begin{array}{l}\text { Bulk } \\
\text { imprinting }\end{array}$ & Haemoglobin & Hydrophilicity & $\begin{array}{l}\text { Polyacrylamide- } \\
\text { chitosan beads } \\
\text { complex }\end{array}$ & {$[13]$} \\
\cline { 2 - 5 } & Lysozyme & $\begin{array}{l}\text { Metal coordination } \\
\text { interaction }\end{array}$ & Hydrogel & {$[24]$} \\
\cline { 2 - 5 } & $\begin{array}{l}\text { Bovine serum albumin, } \\
\text { urease, haemoglobin }\end{array}$ & $\begin{array}{l}\text { Ionic interaction } \\
\text { and hydrogen bonding }\end{array}$ & Polysiloxane & {$[26]$} \\
\hline
\end{tabular}




\begin{tabular}{|c|c|c|c|c|}
\hline & and myoglobin & & & \\
\hline \multirow[t]{3}{*}{$\begin{array}{l}\text { Surface } \\
\text { imprinting }\end{array}$} & $\begin{array}{l}\text { Lysozyme, ribonuclease } \\
\text { A and myoglobin }\end{array}$ & $\begin{array}{l}\text { Hydrophobicity and } \\
\text { charge }\end{array}$ & Polymethacrylate & [33] \\
\hline & Haemoglobin & $\begin{array}{l}\text { Hydrophobicity and } \\
\text { charge }\end{array}$ & $\begin{array}{l}\text { Polysiloxane-silica } \\
\text { complex }\end{array}$ & {$[34]$} \\
\hline & $\begin{array}{l}\text { Horse spleen ferritin } \\
\text { and bovine serum } \\
\text { albumin }\end{array}$ & $\begin{array}{l}\text { Electrostatic forces } \\
\text { and steric effects }\end{array}$ & $\begin{array}{l}\text { Ternary lipid } \\
\text { monolayer }\end{array}$ & [35] \\
\hline \multirow{3}{*}{$\begin{array}{l}\text { Epitope } \\
\text { imprinting }\end{array}$} & Short peptides & Hydrogen bonding & Polyacrylamide & {$[41]$} \\
\hline & $\begin{array}{l}\text { C-terminal protected } \\
\text { phosphorylated short } \\
\text { peptide (Fmoc- } \\
\text { Tyr(PO3H2)-Pro-OH) }\end{array}$ & $\begin{array}{l}\text { Ionic hydrogen } \\
\text { bonding }\end{array}$ & Polymethacrylmide & $\begin{array}{l}\text { Ge, Y. } \\
\text { and } \\
\text { Stein } \\
\text { ke, } \\
\text { J.H.G } \\
\text {., } \\
\text { unpu } \\
\text { blishe } \\
\text { d }\end{array}$ \\
\hline & $\begin{array}{l}\mathrm{N} \text { - and C-terminal } \\
\text { protected } \\
\text { phosphorylated } \\
\text { tyrosine }\end{array}$ & Hydrogen bonding & Polymethacrylate & [46] \\
\hline
\end{tabular}

Table 1. Selected examples of protein imprinting by three main approaches

Bulk imprinting. The most straightforward approach for protein imprinting is bulk imprinting. The advantage of this approach is that the whole protein can be removed through either extraction or washing and is then able to rebind into the three-dimensional cavity within the MIP. Owing to the large size of proteins, controlling the size and number of pores that are generated during the synthesis and spread onto the MIP surface, together with the density of MIP network, is essential for effective MIP design and synthesis.

Polyacrylamides and their derivatives are among the most extensively used polymeric materials for bulk imprinting of proteins. Recent examples include haemoglobin ( $\mathrm{Hb})$ [13], lysozyme [14-16] and bovine serum albumin (BSA) [15-19] imprinted polyacrylamides. However, these polymers usually sacrifice density of the polymer network to facilitate protein transfer. It is well known that low density MIPs are less stable [20], and therefore there is a demand for other more suitable matrix materials, such as hydrogels and sol-gels.

Hydrogels are cross-linked, three-dimensional hydrophilic polymer networks that swell upon contact with water but do not dissolve. They can change volume in response to external factors such as $\mathrm{pH}$ and temperature, and this provides a means to control the polymer network. For instance, the capacity of BSA-imprinted calcium alginate to adsorb BSA was increased with additional hydroxyethyl cellulose, a hydrophilic cellulose derivative [21]. This system had a denser polymer network, decreased network mesh size and increased stability of cross-linkages. These factors were thought to make the big difference by creating more imprinted moieties and thus making the interaction sites less movable and the binding cavities more complementary to the original templates. The syntheses of $\mathrm{Hb}[22,23]$ and lysozyme [24] imprinted hydrogels have also been recently reported.

Sol-gels are another group of advanced polymeric materials applied to bulk imprinting of proteins. They are colloidal suspensions of silica particles that are gelled to form a solid. The advantages of water involvement and the mild condition (i.e. $\mathrm{pH}$ and ionic strength) in the sol-gel process have been exploited for BSA, $\mathrm{Hb}$, myoglobin $(\mathrm{Mb})$ and urease imprinting [25,26]. Recently, Tao et al. [27] described an ovalbumin-imprinted polymer using xero-gel, a sol-gel-derived product that exhibits remarkable stability over time. In addition, its physicochemical properties can be tailored by using different precursors or protocols. A novel strategy for the selective detection and quantification of proteins was established by installing a luminescently tagged molecule within the imprinted site and by monitoring the changes in luminescence upon binding of the analyte. The resulting polymer was 
able to discriminate between the same proteins from different animal species (e.g. human and porcine interleukin-1a).

As discussed above, various approaches are available for the construction of macroporous polymer networks that are used for bulk imprinting and which can entrap and release entire protein molecules. However, the decreased density of some polymers inevitably means that they loose recognition and binding efficiency after a few rounds of regeneration. Also, the pore sizes and functional groups on the polymer surface can change over time under harsh conditions (e.g. high temperature and strong acid). Finally, larger proteins are more difficult to imprint, because it is more problematic to retain selectivity and adsorption with increasing template size. As a result, the alternative approaches of surface imprinting and epitope imprinting have attracted increasing interest.

Surface imprinting. A fundamental strategy of surface imprinting is to locate the imprinted sites at or close to the surface of the MIP, therefore enabling easy access to the target protein molecules. Thus, in comparison with bulk imprinting, the transfer of protein is much easier and the binding kinetic is less restricted although usually lower [28]. The selectivity can also decrease because only part of the protein is bound and recognized. Some elegant examples are discussed below.

A novel surface imprinting approach was introduced by Yilmaz et al. [29], who employed oriented immobilisation of a theophylline template on porous silica beads before polymerisation. After polymerisation, the silica support was dissolved and removed along with the template. This differs from conventional surface imprinting, in which only the template is removed after the synthesis of MIP on a solid support. In subsequent work, amino acids and peptides were also imprinted [30,31]. Inspired by this elegant idea, Li et al. [32] recently developed a surface imprinted polymer nanowire for protein recognition (see Figure 2). Binding and competitive rebinding studies suggested that the high specificity and capacity of the imprinted nanowire was due to the cooperative and multivalent hydrogen bonds between the template and the nanowire. The large surface area of nanowires also resulted in high protein-binding capacity. Furthermore, given that nanowires disperse well in water, they could greatly facilitate applications such as affinity chromatography and biosensing. In addition, Chou and co-workers [33] described a microcontact imprinting method for protein recognition. This approach adapted Yilmaz's idea [29] and, in addition, used a stamping technique. Several advantages were demonstrated, such as rapid MIP synthesis, low amounts of monomers and solvent compatibility of the template.

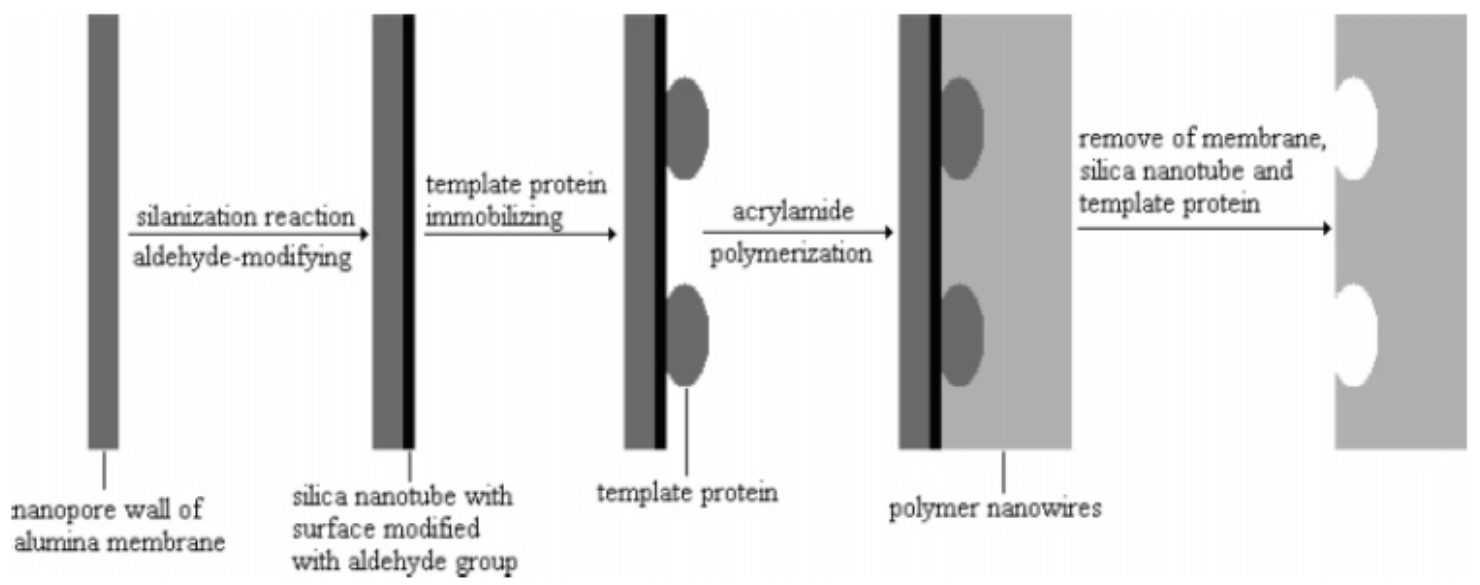

Figure 2. A protein imprinted polymer nanowire employing immobilised template and a sacrificial support. Reprinted with permission from [32]. Copyright 2006 American Chemical Society.

Another recent example of silica-based protein recognition was reported by Shiomi et al. [34]. In their work, the protein template $\mathrm{Hb}$ was first covalently immobilised on the modified silica surface and then removed after a silane layer had been formed on the surface of the silica. Compared with imprinting using free $\mathrm{Hb}$ template, this imprinted silica-silane system proved to be superior in terms of selectivity for $\mathrm{Hb}$ over other proteins (e.g. transferin and chymotrpsinogen A).

It is of particular note that a series of thin, protein-imprinted films or coatings have very recently been applied to the surface of sensors, such as gold electrodes used in quartz crystal microbalances (QCMs) [35-38], and screen-printed platinum electrodes [38]. For example, Zhang et al. [35] reported a novel method that combined sol-gel and self-assembly technology to prepare a thin film imprinted with human serum albumin (HSA) on a gold electrode in a QCM. The resulting sensor showed excellent 
selectivity for the template over other test proteins (e.g. BSA and $\mathrm{Hb}$ ), and this selectivity depended on the overall effect of charge, molecular shape and the degree of swelling of the imprinted film. Rick and Chou [38] used cyclic voltammetric deposition to provide more control of the protein imprinted polymerisation process on a screen-printed platinum support, resulting in enhanced reproducibility of thin polymer films with defined conductivity.

Surface imprinting results in the formation of specific cavities on the surface of the imprinted polymer. These cavities have particular shapes or steric effects that are complementary to parts or fragments of the protein template. However, because of the complexity and conformational flexibility of proteins, the interactions between the protein template and the functional monomer are not as specific and efficient as desired. As a result, protein surface-imprinted polymers sometimes exhibit crossreactivity, non-specific binding and low selectivity. Furthermore, their template capacity is lower than that of protein bulk-imprinted polymers. Hence, an improved and efficient strategy termed 'epitope imprinting' appears to be a very promising way forward, combining the concepts of both surface imprinting and bulk imprinting.

Epitope imprinting. An epitope is the part of a macromolecule that is recognized by the immune system, specifically by antibodies, B cells or cytotoxic T-cells. Inspired by this rational, Rachkov and Minoura [39,40] created a new approach for protein imprinting by imprinting only a short peptide sequence that represents a small and exposed fragment of the whole protein [39,40]. The resulting polymer was able to recognize not only the small peptide template but also the entire protein. This approach was christened 'epitope imprinting'.

Recently, Shea and co-workers [41] presented a remarkable example of epitope imprinting using protein imprinted thin film. They used small peptides fragments of cytochrome c (cyt c) as epitopes to imprint the capture sites (see Figure 3). In comparison with non-imprinted film, the resulting film showed a roughly seven-fold higher affinity and capacity for the target protein. The result of epitope imprinting of BSA showed a significantly high selectivity for BSA when the correct peptide was employed as the epitope. It is of particular note that a very poor selectivity for BSA was observed when a mutant peptide (only one amino acid had been mutated) was used as the epitope under the same conditions. Moreover, non-specific binding was reduced, owing to the low number of potential binding sites for non-target protein, and specific binding was enhanced by cooperative and multivalent interactions, such as hydrogen bonds and hydrophobic interactions. 


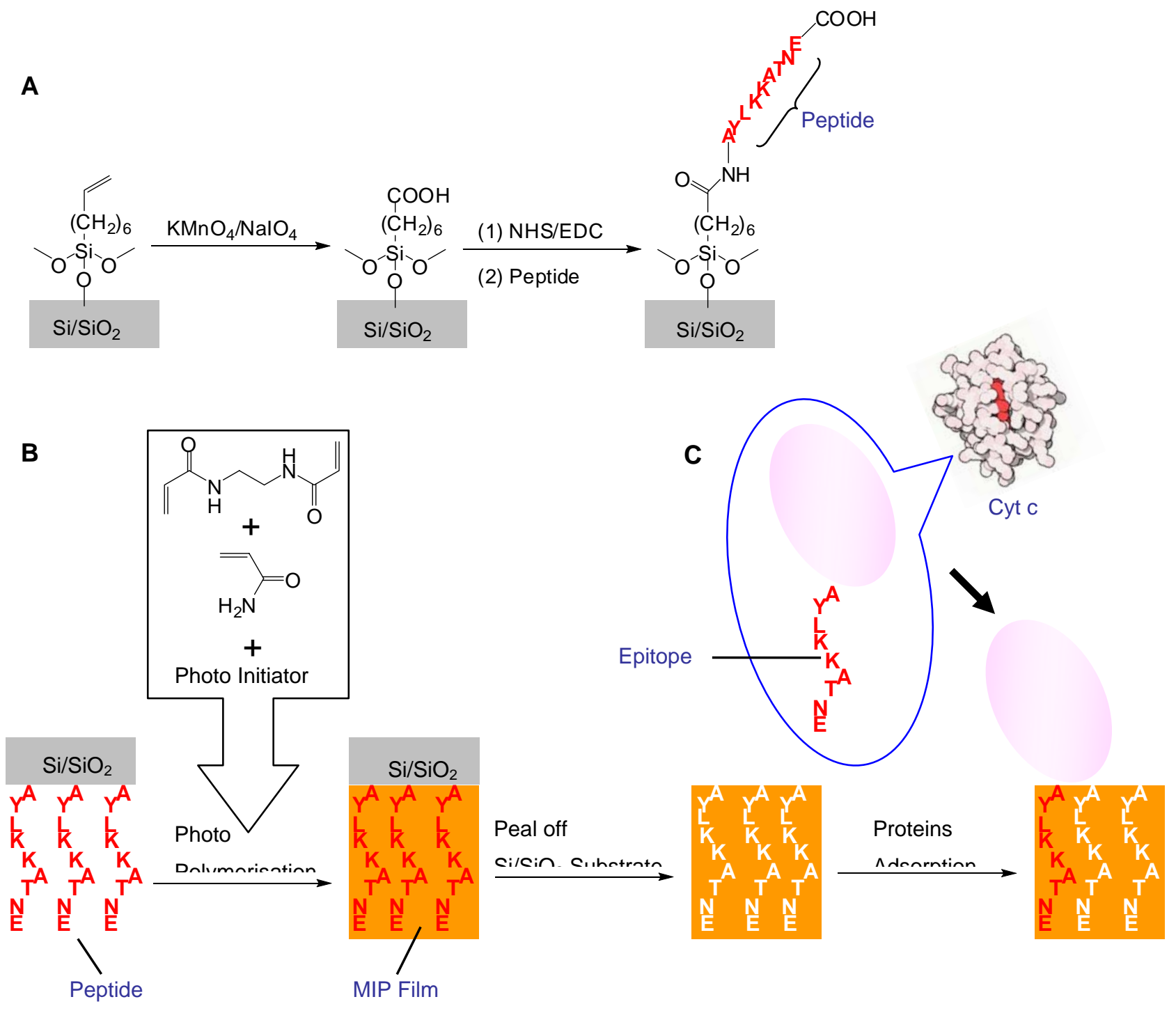

Figure 3. Epitope imprinting using protein imprinted thin film. (a) Method for glass modification and peptide attachment. The vinylsilane surface was oxidized with $\mathrm{KMnO} 4, \mathrm{NaIO} 4$ and $\mathrm{K} 2 \mathrm{CO} 3$. The carboxylic acid-terminated monolayer was activated with a mixture of N-hydroxysuccinimide (NHS) and 1-ethyl-3-(3-dimethylaminopropyl)carbodiimide (EDC). The peptide epitopes were subsequently attached to the substrate in a solution of phosphate buffer ( $\mathrm{PH}$ 7.3) and $\mathrm{NaCl}$. The attachment protocol is illustrated only for N-terminal attachment. (b) Illustration outlining MIP film fabrication and evaluation. The C-terminal nonapeptide epitope is attached through a tether to a glass or oxidized silicon surface by the N-terminal amino acid of the peptide. Monomers were photochemically crosslinked and the glass substrate and/or silicon wafer was removed by soaking in buffer overnight. (c) Proposed mechanism for recognition of C-terminal peptide-sequence-imprinted surfaces. A nanocavitybound target protein is formed by many cooperative weak interactions, involving hydrogen bonds and hydrophobic interactions. Adapted with permission from [41].

Encouraged by the development of a series of urea-based functional monomers [42-44] and their successful application for the recognition of phosphate and phosphorous esters [45], Steinke's group synthesized a new MIP system for the recognition of phosphorylated peptides and proteins by epitope imprinting (Ge, Y. and Steinke, J.H.G., unpublished). Instead of urea-based functional monomers, thiourea-based compounds were used as functional monomers in order to provide stronger ionic hydrogen bonding with phosphate groups, which would result in high affinity and good selectivity. 
An unusual approach for the separation and recognition of cyt c (surface histidine-exposed protein) employed L-histidine as an epitope template, which had metal-coordination interaction with the metalchelating monomer $\mathrm{N}$-methacryloyl-(L)-histidine- $\mathrm{Cu}_{2+}$ [46]. As previously discussed by Conrad II and Shea [20], the metal coordination force enhanced the binding affinity and selectivity. The imprinted system was stable and reusable without considerable loss of template adsorption capacity after the template was removed, but this system is only applicable for certain protein templates (e.g. $\mathrm{Hb}$ and $\mathrm{Mb}$ ) containing exposed residues that can form a metal coordination bond with the monomer.

Epitope imprinting has several advantages. First, more specific and stronger interactions with a small part or fragment of the protein can minimise non-specific binding and improve affinity. Second, organic solvents can be employed for the polymerisation process, because small peptide templates are more stable in these solvents. Finally, synthesizing the short peptides that serve as epitopes is inexpensive. However, modified and functionalised peptides such as phosphorylated peptides are difficult to purify or synthesize.

\section{Other biomacromolecular imprinting}

In addition to proteins, other biomacromolecules or even microorganisms have recently been used as templates for imprinting. A porous blend of poly(ethylene-co-vinyl alcohol) and dextran in the presence of a-amylase as template resulted in a molecularly imprinted membrane that showed selective recognition features for biomacromolecules such as pepsin, albumin and amyloglucosidase [47]. Escherichia coli-imprinted gel granules synthesized by bulk imprinting were able to distinguish between different types and strains of bacteria by using free-zone electrophoresis [48]. However, surface imprinting is a more practical approach owing to the large dimensions of microorganisms, as demonstrated by the successful surface imprinting of cells and viruses (e.g. yeast cell and tobacco mosaic virus) by Dickert et al. [49-51].

\section{Synthetic macromolecular imprinting}

In biomacromolecular imprinting, several physical and chemical compromises need to be made in terms of solubility, stability and identification of functional groups, and it is usually difficult to synthesize the MIPs in an environment that reflects a biological environment. The complexity, inconsistency and expense of some biomacromolecules also hinders its development. Consequently, synthetic macromolecules, particularly those with biomimetic functionalities and defined functional groups such as dendrimers and hyperbranched polymers (HBPs), offer interesting alternative templates and synthetic analogues to biomacromolecules.

\section{Dendrimer and hyperbranched polymer imprinting}

Dendrimers are well defined, three-dimensional and nanoscopic macromolecules constructed from an interior core, with a regular array of branching units. They are able to mimic not only the size and shape of natural macromolecule such as proteins but also biological functions such as molecular recognition of substrates, either in their interior or on the surface [52]. Therefore, they have been increasingly used for a wide range of biological applications such as molecular imaging and drug delivery [52-54]. Furthermore, some dendrimers can be easily degraded under certain conditions (e.g. high or low extremes of $\mathrm{pH}$ ) and dissolved in a range of solvents, which considerably facilitates the process of template removal.

Following some preliminary studies using poly(propylene)imine dendrimer as a single-molecule template for the casting of mesoporous cavities in silica sol-gels [55,56], a remarkably elegant imprinting approach, wherein a single template was dynamically imprinted in a single dendrimer, was recently reported by Zimmerman et al. [57-59]. The significant advantages of this technology were as follows: (i) nearly homogeneous binding sites; (ii) only one binding cavity per polymer molecule; (iii) complete template removal; and (iv) good solubility of the resulting material in common organic solvents. However, technically this example does not constitute dendrimer imprinting, because the removable template was not a dendrimer.

The first genuine examples of dendrimer imprinting were presented by Sellergren and Hall*. In addition to dendrimer imprinting, they also introduced hyperbranched polymer (HBP) imprinting for the first time*. Unlike well defined and monodispersed dendrimers, HBPs are polydispersed and have defects in the form of built-in linear segments; but they are they are still highly branched dendritic molecules. HBPs are promising and competitive materials owing to their relative ease of preparation and cost-effectiveness [60]. Various HBPs have been investigated and have proved competitive with dendrimers for biological applications such as device delivery [61] and as oxygen carriage [62]. In common with dendrimers, HBPs can be partially or completely decomposed under the right conditions and have good solubility in a range of solvents, facilitating temple removal. 
In Sellergren and Hall's patent application*, MIP was synthesized using either a dendrimer or HBP as the template with at least one functional monomer (e.g. carboxylic acid, boronate or urea functional monomer) and through either bulk imprinting or surface imprinting. After the template was removed, the resulting MIP was used for affinity-phase separation or sensing of relevant proteins, polynucleotides or polysaccharides, which have similar size, shape and functional groups to the polymeric template. Owing to the multifunctionality of the terminal groups on the surface of the dendrimer and HBP, the properties of the complementary domain of MIP can be modified and specially designed. For instance, when some small ligands such as peptides and oligosugars are attached to the surface of a dendrimer or HBP template, additional imprinted sites are created, thus mimicking functionalised biomacromolecules (e.g. glycosylated $\mathrm{Hb}$ imprinting). Furthermore, combined with other pore-forming species, such as silane-modified silica and porous silica, dendrimers and HBPs could be either used as templates to increase accessibility of MIP binding cavities or attached to porous disposable solid supports for subsequent MIP synthesis by adapting Yilmaz's method [29].

In conclusion, synthetic macromolecular imprinting using dendrimers and HBPs is a promising approach for a broad range of applications. An important advantage of this technology is that MIPs can be synthesized and analysed in either non-aqueous or aqueous solutions without conformational change or loss of bioactivity of the templates, which is an obstacle to most types of biomacromolecular imprinting. In addition, this technique could facilitate a range of highly functionalised and reactive macro-templates that are either not found in nature or difficult to obtain. It should be noted, however, that the expense and laborious synthesis of dendrimers and the high polydispersity of HBPs might restrict the widespread use of this technology.

\section{Conclusions and outlook}

The field of macromolecular imprinting has expanded rapidly in recent years, particularly owing to the considerable increase in demand for macromolecular detection, separation and analysis. There are multifarious examples of successful molecular imprinting for relatively low molecular weight molecules, such as steroids and pesticides. In addition, large biomolecules such as proteins and cells have also been successfully imprinted. However, because of their natural limitations, conventional preparation and analysis of imprinted polymers has to be modified to accommodate the physical properties (e.g. solubility, stability and conformation) of these biotemplates. Therefore, synthetic analogues, such as dendrimers and HBPs, could provide alternative and practical templates for future imprinting applications. Meanwhile, among the three general approaches for macromolecular imprinting, epitope imprinting seems be advantageous, owing to its desirable characteristics, such as increased specific recognition and higher affinity. However, more investigations are required to further improve selectivity, stability, site accessibility and cost-effectiveness, especially in biomedical applications such as medical devices, diagnostics, drug delivery and biological assays. Excellent progress has been made, but it might be some time before a generic and scaleable approach to imprint macromolecules will be achieved. 


\section{References}

1 Komiyama, M. et al. eds (2003) Molecular Imprinting, Wiley-VCH Verlag GmbH \& Co. KGaA, Weinheim

2 Haupt, K. (2003) Molecularly imprinted polymers: the next generation. Anal. Chem. 9, 376A-383A

3 Yan, M. and Ramström, O. eds (2005) Molecularly imprinted materials-science and technology, 1st edn. Marcel Dekker, New York

4 Mahony, J.O. et al. (2005) Molecularly imprinted polymers - potential and challenges in analytical chemistry. Anal. Chim. Acta 534, 31-39

5 Hall, A.J. et al. (2005) Imprinted polymers. In Topics in Current Chemistry 249: Templates in Chemistry II (Schalley, C.A., Vögtle, F. and Dötz K.H., eds), pp. 317-349, Springer Berlin/Heidelber

6 Piletsky, S.A. and Turner, A.P.F., eds (2006) Molecular imprinting of polymers, Landes Bioscience, Georgetown, Texas

7 Wei, S. et al. (2006) Capturing molecules with templated materials - analysis and rational design of molecularly imprinted polymers. Anal. Chim. Acta 578, 50-58

8 Nicholson, J.W. (2006) The Chemistry of Polymers ( $3_{\mathrm{rd}}$ edn), The Royal Society of Chemistry Publishing

9 Hilt, J.Z. and Byrne, M.E. (2004) Configurational biomimesis in drug delivery: molecular imprinting of biologically significant molecules. Adv. Drug Deliv. Rev. 56, 1599-1620

10 Turner, N.W. et al. (2006) From 3D to 2D: a review of the molecular imprinting of proteins. Biotechnol. Prog. 22, 1474-1489

11 Bossi, A. et al. (2007) Molecularly imprinted polymers for the recognition of proteins: the state of art. Biosens. Bioelectron. 22, 1131-1137

12 Ramström, O. and Ansell, R.J. (1998) Molecular imprinting technology: challenges and prospects for the future. Chirality 10, 195-209

13 Guo, T.Y. et al. (2004) Adsorptive separation of hemoglobin by molecularly imprinted chitosan beads. Biomaterials 25, 59055912

$14 \mathrm{Ou}$, S.H. et al. (2004) Polyacrylamide gels with electrostatic functional groups for the molecular imprinting of lysozyme. Anal. Chim. Acta 504, 163-166

15 Huang, J.T. et al. (2005) Template imprinting amphoteric polymer for the recognition of proteins. J. Appl. Polym. Sci. 95, $358-361$

$16 \mathrm{Lu}$, S. et al. (2006) Protein-imprinted soft-wet gel composite microspheres with magnetic susceptibility II. Characteristics. J. Appl. Polym. Sci. 99, 2401-2407

17 Pang, X. et al. (2005) Bovine serum albumin-imprinted polyacrylamide gel beads prepared via inverse-phase seed suspension polymerization. Anal. Chim. Acta 550, 13-17

18 Pang, X. et al. (2006) Soft-wet polyacrylamide gel beads with the imprinting of bovine serum albumin. React. \& Func. Polym. $66,1182-1188$

19 Pang, X. et al. (2006) Synthesis of polyacrylamide gel beads with electrostatic functional groups for the molecular imprinting of bovine serum albumin. Anal. Bioanal. Chem. 384, 225-230

20 Conrad, P.G., II and Shea, K.J. (2005) Use of metal co-ordination for controlling imprinted polymers. In Molecularly Imprinted Materials: Science and Technology (1 $1_{\mathrm{st}}$ edn) (Yan, M. and Ramström, O., eds), pp. 123-180, Marcel Dekker, New York

21 Zhang, F. et al. (2006) Emulsion and macromolecules templated alginate based polymer microspheres. React. \& Func. Polym. $66,712-719$

22 Xia, Y. et al. (2005) Hemoglobin recognition by imprinting in semi-interpenetrating polymer network hydrogel based on polyacrylamide and chitosan. Biomacromolecules 6, 2601-2606

23 Hawkins, D.M. et al. (2006) Quantification and confocal imaging of protein specific molecularly imprinted polymers. Biomacromolecules 7, 2560-2564

24 Odabşi, M. et al. (2007) Molecular imprinted particles for lysozyme purification. Mater. Sci. \& Engin. C 27, 90-99

25 Venton, D.L. and Gudipati, E. (1995) Entrapment of enzymes using organo-functionalized polysiloxane copolymers. Biochim. Biophys. Acta 1250, 117-125

26 Venton, D.L. and Gudipati, E. (1995) Influence of protein on polysiloxane polymer formation: Evidence for induction of complementary protein-polymer interactions. Biochim. Biophys. Acta 1250, 126-136

27 Tao, Z. et al. (2006) Templated xerogels as platforms for biomolecule-less biomolecule sensors. Anal. Chim. Acta 564, 59-65

28 Bossi, A. et al. (2001) Surface-grafted molecularly imprinted polymers for protein recognition. Anal. Chem. 73, 5281-5286

29 Yilmaz, E. et al. (2000) The use of immobilized templates - A new approach in molecular imprinting. Angew. Chem. Int. Ed. Engl. 39, 2115-2118

30 Titirici, M.M. et al. (2002) Hierarchically imprinted stationary phases: Mesoporous polymer beads containing surfaceconfined binding sites for adenine. Chem. Mater. 14, 21-23

31 Titirici, M.M. et al. (2003) Hierarchical imprinting using crude solid phase peptide synthesis products as templates. Chem. Mater. 15, 822-824

$32 \mathrm{Li}$, Y. et al. (2006) Protein recognition via surface molecularly imprinted polymer nanowires. Anal. Chem. 78, 317-320

33 Lin, H.Y. et al. (2006) The microcontact imprinting of proteins: The effect of cross-linking monomers for lysozyme, ribonuclease A and myoglobin. Biosens. Bioelectron. 22, 534-543

34 Shiomi, T. et al. (2005) A method for the molecular imprinting of hemoglobin on silica surfaces using silanes. Biomaterials 26, $5564-5571$

35 Zhang, Z. et al. (2006) Molecularly imprinted thin film self-assembled on piezoelectric quartz crystal surface by the sol-gel process for protein recognition. Biosens. Bioelectron. 21, 1244-1251

36 Turner, N. et al. (2007) Formation of protein molecular imprints within Langmuir monolayers: A quartz crystal microbalance study. J. Colloid Interface Sci. 308, 71-80

37 Rick, J. and Chou, T.C. (2005) Imprinting unique motifs formed from protein-protein associations. Anal. Chim. Acta 542, 2631

38 Rick, J. and Chou, T.C. (2006) Using protein templates to direct the formation of thin-film polymer surfaces. Biosens. Bioelectron. 22, 544-549

39 Rachkov, A. and Minoura, N. (2001) Towards molecularly imprinted polymers selective to peptides and proteins. The epitope approach. Biochim. Biophys. Acta 1544, 255-266

40 Rachkov, A. et al. (2004) Molecularly imprinted polymers prepared in aqueous solution selective for [Sar(1), Ala(8)]angiotensin II. Anal. Chim. Acta 504, 191-197

41 Nishino, H. et al. (2006) Selective protein capture by epitope imprinting. Angew. Chem. Int. Ed. Engl. 45, 2392-2396

42 Hall, A.J. et al. (2005) Urea host monomers for stoichiometric molecular imprinting of oxyanions. J. Org. Chem. 70, 17321736

43 Manesiotis, P. et al. (2005) Improved imide receptors by imprinting using pyrimidine-based fluorescent reporter monomers. 
J. Org. Chem. 70, 2729-2738

44 Urraca, J.L. et al. (2006) A stoichiometric molecularly imprinted polymer for the class-selective recognition of antibiotics in aqueous media. Angew. Chem. Int. Ed. Engl. 45, 5158-5161

45 Hall, A.J. et al. (2005) Non-covalent imprinting of phosphorous esters. Anal. Chim. Acta 538, 9-14

46 Özcan, A.A. et al. (2006) L-histidine imprinted synthetic receptor for biochromatography applications. Anal. Chem. 78, 72537258

47 Silvestri, D. et al. (2006) Molecularly imprinted membranes for an improved recognition of biomolecules in aqueous medium. J. Memb. Sci. 282, 284-295

48 Bacskay, I. et al. (2006) Universal method for synthesis of artificial gel antibodies by the imprinting approach combined with a unique electrophoresis technique for detection of minute structural differences of proteins, viruses, and cells (bacteria). III: Gel antibodies against cells (bacteria). Electrophoresis 27, 4682-4687

49 Dickert, F.L. and Hayden, O. (2002) Bioimprinting of polymers and sol-gel phases. Selective detection of yeasts with imprinted polymers. Anal. Chem. 74, 1302-1306

50 Hayden, O. et al. (2003) Mass-sensitive detection of cells, viruses and enzymes with artificial receptors. Sens. Actuators B Chem. 91, 316-319

51 Hayden, O. et al. (2006) Artificial antibodies for bioanalyte detection-sensing viruses and proteins. Adv. Funct. Mater. 16, $1269-1278$

52 Helms, B. and Meijer, E.W. (2006) Dendrimers at work. Science 313, 929-930

53 Yang, H. and Kao, W.J. (2006) Dendrimers for pharmaceutical and biomedical applications. J. Biomater Sci Poly Ed. 17, 3-19

54 Svenson, S. and Tomalia, D.A. (2005) Dendrimers in biomedical applications - reflections on the field. Adv. Drug Deliv. Rev. $57,2106-2129$

55 Larsen, G. et al. (2000) Facile sol-gel synthesis of porous silicas using poly(propylene)imine dendrimers as templates. J. Mater. Res. 15, 1842-1848

56 Larsen, G. and Noriega, S. (2004) Dendrimer-mediated formation of $\mathrm{Cu}-\mathrm{CuOx}$ nanoparticles on silica and their physical and catalytic characterization. Appl. Cata A: General 278, 73-81

57 Zimmerman, S.C. et al. (2002) Synthetic hosts by monomolecular imprinting inside dendrimers. Nature 418, 399-403

58 Mertz, E. and Zimmerman, S.C. (2003) Cross-linked dendrimer hosts containing reporter groups for amine guests. J. Am. Chem. Soc. $125,3424-3425$

59 Zimmerman S.C. and Lemcoff N.G. (2004) Synthetic hosts via molecular imprinting - are universal synthetic antibodies realistically possible? Chem. Commun. (Camb.) 5-14, DOI:10.1039/b304720b

60 Gao, C. and Yan, D. (2004) Hyperbranched polymers: from synthesis to applications. Prog. Polym. Sci. 29, 183-275

61 Yates, C.R. and Hayes, W. (2004) Synthesis and applications of hyperbranched polymers. Euro. Poly. J. 40, 1257-1281

62 Twyman, L.J. and Ge, Y. (2006) Porphyrin cored hyperbranched polymers as heme protein models. Chem. Commun. (Camb.) $15,1658-1660$

* Sellergren, B. and Hall, A.J. (2006) Imprinting using dendrimers as templates. Publication No. WO/2006/041370; International Patent No. (PCT/SE2005/001330). Patent application awaiting approval. 